\title{
A Semi-supervised Manifold Alignment Algorithm and an Evaluation Method Based on Local Structure Preservation
}

\author{
Xiaojie $\mathrm{Li}^{1}$, Jiancheng $\mathrm{Lv}^{2 *}, \mathrm{Xi} \mathrm{Wu}^{1}$, and $\mathrm{Xin} \mathrm{Yu}^{3}$ \\ 1.College of Computer Science, Chengdu University of Information Technology \\ 2. College of Computer Science, Sichuan University \\ 3.State Key Laboratory of Computer Architecture, Institute of Computing Technology, \\ CAS, China \\ ${ }^{*}$ Corresponding author: Jiancheng Lv e-mail: lvjiancheng@scu.edu.cn.
}

\begin{abstract}
Manifold alignment is the process of aligning the shared intrinsic structure extracted from multiple manifolds. The criteria of manifold alignment are 2-fold. First, the alignment should minimize the distance between manifolds, ensuring high alignment accuracy. Second, the original structure should be preserved. Currently, most alignment algorithms focus on alignment accuracy, whereas structure preservation has received little attention. This paper proposes a new semi-supervised alignment method that combines locally linear reconstructions in each manifold. The shared intrinsic structure is obtained by solving an optimization problem with a closed-form solution, which simultaneously matches the corresponding instances and preserves the local geometry of each manifold. Furthermore, a new method is presented to evaluate the local structure preservation. The structural stability is defined as a metric of the local structure preservation property. It is shown that the proposed method preserves the local geometry of the original dataset well. It is also found that better structural stability gives higher alignment accuracy, but the converse is not true. Experimental results on both synthetic and real datasets demonstrate the effectiveness and efficiency of our method.
\end{abstract}

Keywords: Manifold alignment, structural stability, local structure 


\section{Introduction}

Many areas of machine learning, information retrieval, and computer vision consider multiple high-dimensional datasets that contain a shared intrinsic structure. As different datasets might be represented by different features, it is difficult to align multiple datasets in their original high-dimensional spaces. Thus, extracting the shared intrinsic structure from multiple highdimensional datasets becomes increasingly important $[1,2,3,4,5]$. Manifold alignment extracts the shared intrinsic structure, allowing multiple datasets to be aligned in a low-dimensional space.

Over the past decade, many linear and nonlinear dimensionality reduction approaches have been developed to extract the intrinsic structure from a dataset, e.g., sparse manifold clustering and embedding $[4,6,7]$, multidimensional scaling [8, 9], isometric mapping [10], locally linear embedding [11, 12], Laplacian eigenmaps [13], principal component analysis [14, 15, 16] and locally preserving projection $[17,18]$. However, these common dimensionality reduction methods cannot be directly used to align multiple datasets[19]. This paper focuses on aligning multiple datasets by extracting their shared intrinsic structure.

Currently, there exist a number of manifold alignment methods $[1,2,3,20$, 21, 22, 23, 24, 25, 26, 27, 28, 29]. Ham et al. [3] proposed a semi-supervised manifold alignment algorithm, where feature constraints were imposed on the graph Laplacian process. Hoa et al. [20] presented a framework for aligning two sequentially-ordered datasets. In [1], Wang et al. proposed an important framework for unsupervised alignment. In addition, Wang and Mahadevan found an optimal rotation and scaling transformation to align pre-embedded instances [2]. Pei et al. proposed a nonrigid alignment based on a parameterized distance curve matrix [24].

However, manifold alignment has two criteria [1, 24, 30]. First, the alignment should minimize the distance between manifolds, so that high alignment accuracy can be obtained. Second, the alignment should not destroy the original structure of the datasets. Almost all existing manifold alignment methods focus on the former $[2,24,31,32,33]$. Structure preservation has received little attention $[3,34,35,18]$. Furthermore, there are no evaluation metrics to evaluate structure preservation techniques.

This paper proposes a new semi-supervised alignment method by combining a locally linear reconstruction in each manifold, and presents a new method for the evaluation of local structure preservation. The shared intrin- 
sic structure is obtained by solving an optimization problem with a closedform solution, which simultaneously matches the corresponding instances and preserves the local geometry of each manifold. This paper defines the structural stability as a metric of the local structure preservation property $[36,37,38]$. It is shown that the proposed method effectively preserves the local geometry of the original dataset. It is also seen that better structural stability leads to higher alignment accuracy, but the converse is not true. Experimental results on both synthetic and real datasets demonstrate the effectiveness and efficiency of our method.

The remainder of this paper is organized as follows. Section 2 describes the generic problem of manifold alignment and presents some preliminary results. Our manifold alignment method is proposed in Section 3, and Section 4 introduces the new method for evaluating the local structure preservation. In Section 5, experimental results on both synthetic and real datasets demonstrate the effectiveness and efficiency of our method. Finally, some conclusions are drawn in Section 6.

\section{The problem of manifold alignment}

Given two manifolds parameterized by the same intrinsic structure:

$$
\left\{\begin{array}{l}
\mathbf{X}=\left[\mathbf{x}_{1}, \mathbf{x}_{2}, \cdots, \mathbf{x}_{m}\right] \in R^{D 1 \times m} \\
\mathbf{Y}=\left[\mathbf{y}_{1}, \mathbf{y}_{2}, \cdots, \mathbf{y}_{n}\right] \in R^{D 2 \times n}
\end{array}\right.
$$

where each column vector $\mathbf{x}_{i} \in R^{D 1}\left(\mathbf{y}_{j} \in R^{D 2}\right)$ denotes an instance in the original high-dimensional space, $D 1$ ( $D 2)$ denotes the number of features or dimensions in the manifold, and $m(n)$ indicates the number of data points in the manifold. Suppose $m \leq n$. A corresponding pair, denoted by $\left(\mathbf{x}_{i} \leftrightarrow \mathbf{y}_{j}\right)$, means that $\mathbf{x}_{i}$ and $\mathbf{y}_{j}$ have the same latent features. With some correspondence constraints, we wish to map $\mathbf{X}, \mathbf{Y}$ to $\mathbf{F}_{\mathbf{X}}=\left\{F_{\mathbf{x}_{1}}, F_{\mathbf{x}_{2}}, F_{\mathbf{x}_{m}}\right\} \in R^{d \times m}$, $\mathbf{F}_{\mathbf{Y}}=\left\{F_{\mathbf{y}_{1}}, F_{\mathbf{y}_{2}}, F_{\mathbf{y}_{n}}\right\} \in R^{d \times n}$ such that if $\left(\mathbf{x}_{i} \leftrightarrow \mathbf{y}_{j}\right)$, their locations in the shared intrinsic space, $\mathbf{F}_{\mathbf{x}_{i}}$ and $\mathbf{F}_{\mathbf{y}_{j}}$, should be as close together as possible, where $d$ denotes the dimension of the shared intrinsic space.

After manifold alignment, the closest pairs across two manifolds are viewed as corresponding pairs in the shared intrinsic space. Given $\mathbf{F}_{\mathbf{x}_{i}}(i=1,2, \cdots, m)$, if

$$
c=\arg \min \left\|\mathbf{F}_{\mathbf{x}_{i}}-\mathbf{F}_{\mathbf{y}_{j}}\right\| \quad j=1,2, \cdots, n,
$$


then $\left(\mathbf{F}_{\mathbf{x}_{i}} \leftrightarrow \mathbf{F}_{\mathbf{y}_{c}}\right)[1,24,30]$. Clearly, if $\left(\mathbf{x}_{i} \leftrightarrow \mathbf{y}_{c}\right), \mathbf{F}_{\mathbf{x}_{i}}$ should be closer to $\mathbf{F}_{\mathbf{y}_{c}}$ in terms of Euclidean distance. $\mathbf{F}_{\mathbf{x}_{i}}=\mathbf{F}_{\mathbf{y}_{c}}$ if and only if instances $\mathbf{x}_{i}$ and $\mathbf{y}_{c}$ are in exact correspondence [1].

To map $\mathbf{X}, \mathbf{Y}$ to $\mathbf{F}_{\mathbf{X}}, \mathbf{F}_{\mathbf{Y}}$, several alignment methods have been proposed. However, regardless of the mapping of $\mathbf{X}, \mathbf{Y}$ to $\mathbf{F}_{\mathbf{X}}, \mathbf{F}_{\mathbf{Y}}$ the alignment method should not destroy the original structure. In this paper, a new semi-supervised alignment is proposed. The shared intrinsic structure is obtained by solving an optimization problem with a closed-form solution, which simultaneously matches the corresponding instances and preserves the local geometry of each manifold. Furthermore, a new method is presented for the evaluation of the local structure preservation.

\section{Semi-supervised manifold alignment based on locally linear re- construction}

The proposed Semi-supervised alignment algorithm based on Locally Linear Reconstruction is introduced in this section (named SLLR). We proceed by formulating an optimization problem with a closed-form solution.

\subsection{Construct the Sparse Weight Matrix of Each Manifold}

It is important to construct a weight matrix describing the relationship among samples. Of the many approaches that have been reported, the $l_{1}$ method [39] is often used to form this matrix. In our experiments, the knearest neighbor method is employed to construct the sparse weight matrix of each manifold [40], i.e., $W_{\mathbf{x}} \in R^{m \times m}$ and $W_{\mathbf{y}} \in R^{n \times n}$.

$$
\begin{aligned}
& \mathbf{W}_{\mathbf{x}}\left\{\begin{array}{l}
W_{\mathbf{x}(i, j)}=e^{-\left\|\mathbf{x}_{i}-\mathbf{x}_{j}\right\|^{2} / \theta^{2}} \quad \text { if } \mathbf{x}_{j} \text { is among the } \mathrm{k} \\
\text { nearest neighbors of } \mathbf{x}_{i}(i, j=1,2, \cdots, m ; i \neq j), \\
W_{\mathbf{x}(i, j)}=0 \quad \text { otherwise. }
\end{array}\right. \\
& \mathbf{W}_{\mathbf{y}}\left\{\begin{array}{l}
W_{\mathbf{y}_{(i, j)}}=e^{-\left\|\mathbf{y}_{i}-\mathbf{y}_{j}\right\|^{2} / \theta^{2}} \quad \text { if } \mathbf{y}_{j} \text { is among the } \mathrm{k} \\
\text { nearest neighbors of } \mathbf{y}_{i}(i, j=1,2, \cdots, n ; i \neq j), \\
W_{\mathbf{y}_{(i, j)}}=0 \quad \text { otherwise. }
\end{array}\right.
\end{aligned}
$$




\subsection{Energy Function Definition}

Suppose $\mathcal{S}$ is a set of prior corresponding pairs:

$$
\mathcal{S}=\left\{\left(\mathbf{x}_{1} \leftrightarrow \mathbf{y}_{1}\right),\left(\mathbf{x}_{2} \leftrightarrow \mathbf{y}_{2}\right), \cdots,\left(\mathbf{x}_{s} \leftrightarrow \mathbf{y}_{s}\right)\right\}
$$

where $s=\operatorname{Card}(\mathcal{S})$ denotes the number of elements of the set $\mathcal{S}$ and $s<m$. The correspondence matrix $\mathbf{W}^{\mathbf{x y}} \in R^{m \times n}$ is then constructed as:

$$
\mathbf{W}^{\mathbf{x y}} \begin{cases}W_{(i, j)}^{\mathbf{x y}}=1 & \text { if }\left(\mathbf{x}_{i} \leftrightarrow \mathbf{y}_{j}\right) \in S, \\ W_{(i, j)}^{\mathbf{x y}}=0 & \text { otherwise. }\end{cases}
$$

Semi-supervised manifold alignment is achieved by minimizing the following loss function:

$$
\begin{aligned}
& \mathcal{L}(\mathbf{F})=\mu_{s} \mathbf{E}_{\mathbf{s}}+\mu_{x} \mathbf{E}_{\mathbf{x}}+\mu_{y} \mathbf{E}_{\mathbf{y}}, \\
& \left\{\begin{array}{l}
\mathbf{E}_{\mathbf{s}}=\sum_{\left(\mathbf{x}_{i} \leftrightarrow \mathbf{y}_{j}\right) \in \mathcal{S}}\left\|\mathbf{F}_{\mathbf{x}_{i}}-\mathbf{F}_{\mathbf{y}_{j}}\right\|^{2} W_{(i, j)}^{\mathbf{x y}}, \\
\mathbf{E}_{\mathbf{x}}=\sum_{i}^{m}\left\|\mathbf{F}_{\mathbf{x}_{i}}-\sum_{j}^{m} W_{\mathbf{x}(i, j)} \mathbf{F}_{\mathbf{x}_{j}}\right\|_{2}^{2}, \\
\mathbf{E}_{\mathbf{y}}=\sum_{j}^{n}\left\|\mathbf{F}_{\mathbf{y}_{j}}-\sum_{i}^{n} W_{\mathbf{y}(j, i)} \mathbf{F}_{\mathbf{y}_{i}}\right\|_{2}^{2},
\end{array}\right.
\end{aligned}
$$

where $\mathbf{F}=\left(\mathbf{F}_{\mathbf{X}}, \mathbf{F}_{\mathbf{Y}}\right), \mu_{s}, \mu_{x}, \mu_{y}$ are trade-off parameters.

The first term $\mathbf{E}_{\mathbf{s}}$ addresses the prior corresponding pairs in $\mathcal{S}$. If $\left(\mathbf{x}_{i} \leftrightarrow\right.$ $\left.\mathbf{y}_{j}\right) \in \mathcal{S}, \mathbf{F}_{\mathbf{x}_{i}}$ and $\mathbf{F}_{\mathbf{y}_{j}}$ should be as close as possible to each other in the shared intrinsic space. However, mapping from $\mathbf{X}, \mathbf{Y}$ to $\mathbf{F}_{\mathbf{X}}, \mathbf{F}_{\mathbf{Y}}$ should preserve the original structures. Based on simple geometric intuition, the last two terms, $\mathbf{E}_{\mathbf{x}}$ and $\mathbf{E}_{\mathbf{y}}$, attempt to preserve the local structure of each manifold using the locally linear reconstruction. The low-dimensional embedding computed by both terms has the property that nearby points in the original space remain nearby and similarly co-located with respect to one another in the embedding space [11]. The $l_{2}$ norm is employed to measure the reconstruction error.

\subsection{Optimal Solution}

This section derives the optimal solution to Eq.(2) using the Lagrange multiplier method. The correspondence-preserving term $\mathbf{E}_{\mathbf{s}}$ in Eq.(2) can be rewritten as:

$$
\begin{aligned}
\mathbf{E}_{\mathbf{s}}= & \operatorname{tr}\left(\mathbf{F}_{\mathbf{X}} \mathbf{D}^{\mathbf{x}} \mathbf{F}_{\mathbf{X}}{ }^{T}+\mathbf{F}_{\mathbf{Y}} \mathbf{D}^{\mathbf{y}} \mathbf{F}_{\mathbf{Y}}{ }^{T}\right. \\
& \left.-\mathbf{F}_{\mathbf{X}} \mathbf{W}^{\mathbf{x y}} \mathbf{F}_{\mathbf{Y}}{ }^{T}-\mathbf{F}_{\mathbf{Y}}\left(\mathbf{W}^{\mathbf{x y}}\right)^{T} \mathbf{F}_{\mathbf{X}}{ }^{T}\right),
\end{aligned}
$$


where $\operatorname{tr}($.$) is the trace operator,$

$$
\begin{aligned}
& \mathbf{D}^{\mathbf{x}} \in R^{m \times m}\left\{\begin{array}{l}
D_{(i, i)}^{\mathbf{x}}=\sum_{j}^{n} W_{(i, j)}^{\mathbf{x y}}, \\
D_{(i, j)}^{\mathbf{x}}=0 \text { otherwise, }
\end{array}\right. \\
& \mathbf{D}^{\mathbf{y}} \in R^{n \times n}\left\{\begin{array}{l}
D_{(j, j)}^{\mathbf{y}}=\sum_{i}^{m} W_{(i, j)}^{\mathbf{x y}}, \\
D_{(j, i)}^{\mathbf{y}}=0 \text { otherwise. }
\end{array}\right.
\end{aligned}
$$

Furthermore, we have the matrix forms of $\mathbf{E}_{\mathbf{x}}$ and $\mathbf{E}_{\mathbf{y}}$ :

$$
\left\{\begin{array}{l}
\mathbf{E}_{\mathbf{x}}=\sum_{i}^{m}\left\|\mathbf{F}_{\mathbf{x}_{i}}-\sum_{j}^{m} W_{\mathbf{x}(i, j)} \mathbf{F}_{\mathbf{x}_{j}}\right\|_{2}^{2}=\operatorname{tr}\left(\mathbf{F}_{\mathbf{X}} \mathbf{M}_{\mathbf{x}} \mathbf{F}_{\mathbf{X}}^{T}\right) \\
\mathbf{E}_{\mathbf{y}}=\sum_{j}^{n}\left\|\mathbf{F}_{\mathbf{y}_{j}}-\sum_{i}^{n} W_{\mathbf{y}(j, i)} \mathbf{F}_{\mathbf{x}_{i}}\right\|_{2}^{2}=\operatorname{tr}\left(\mathbf{F}_{\mathbf{Y}} \mathbf{M}_{\mathbf{y}} \mathbf{F}_{\mathbf{Y}}^{T}\right)
\end{array}\right.
$$

where

$$
\left\{\begin{array}{l}
\mathbf{M}_{\mathbf{x}}=\left(\mathbf{I}-\mathbf{W}_{\mathbf{x}}^{T}\right)\left(\mathbf{I}-\mathbf{W}_{\mathbf{x}}\right) \in R^{m \times m}, \\
\mathbf{M}_{\mathbf{y}}=\left(\mathbf{I}-\mathbf{W}_{\mathbf{y}}^{T}\right)\left(\mathbf{I}-\mathbf{W}_{\mathbf{y}}\right) \in R^{n \times n}
\end{array}\right.
$$

Thus, the loss function in Eq.(2) can be rewritten as:

$$
\mathcal{L}(\mathbf{F})=\mu_{s} \mathbf{E}_{\mathbf{s}}+\mu_{x} \mathbf{E}_{\mathbf{x}}+\mu_{y} \mathbf{E}_{\mathbf{y}}=\operatorname{tr}\left(\mathbf{F A F} \mathbf{F}^{T}\right),
$$

where

$$
\mathbf{F}=\left(\mathbf{F}_{\mathbf{X}}, \mathbf{F}_{\mathbf{Y}}\right), \mathbf{A}=\left(\begin{array}{cc}
\mu_{s} \mathbf{D}^{\mathbf{x}}+\mu_{x} \mathbf{M}_{\mathbf{x}} & -\mu_{s} \mathbf{W}^{\mathbf{x y}} \\
-\mu_{s}\left(\mathbf{W}^{\mathbf{x y}}\right)^{T} & \mu_{s} \mathbf{D}^{\mathbf{y}}+\mu_{y} \mathbf{M}_{\mathbf{y}}
\end{array}\right)
$$

To avoid the trivial solution of minimizing $\mathcal{L}(\mathbf{F})$ and make the solution unique with respect to translation, rotation, and scaling, the following constraints are required:

$$
\left\{\begin{array}{l}
\sum_{i=1}^{m+n} \mathbf{f}_{i}=\mathbf{0}, \quad \text { where } \mathbf{f}_{i} \in R^{d \times 1} \\
\frac{1}{(m+n)} \mathbf{F F}^{T}=\mathbf{I}, \quad \text { where } \mathbf{I} \in R^{d \times d}
\end{array}\right.
$$


Since

$$
\begin{aligned}
\operatorname{cov}(\mathbf{F}, \mathbf{F}) & =\sum_{i=1}^{m+n} E\left[\left(\mathbf{f}_{i}-E\left[\mathbf{f}_{i}\right]\right)\left(\mathbf{f}_{i}-E\left[\mathbf{f}_{i}\right]\right)^{T}\right] \\
& =\frac{1}{m+n} \sum_{i=1}^{m+n} \mathbf{f}_{i} \mathbf{f}_{i}^{T} \\
& =\frac{1}{m+n} \mathbf{F F}^{T}=\mathbf{I}
\end{aligned}
$$

and manifold alignment is achieved by minimizing:

$$
\underset{\mathbf{F}}{\arg \min } \mathcal{L}(\mathbf{F})=\operatorname{tr}\left(\mathbf{F A F} \mathbf{F}^{T}\right), \text { s.t., } \frac{1}{(m+n)} \mathbf{F F}^{T}=\mathbf{I} .
$$

The Lagrange multiplier method is employed to solve the optimization problem (5):

$$
\underset{\mathbf{F}}{\arg \min } \operatorname{tr}\left(\mathbf{F A F} \mathbf{F}^{T}\right)+\lambda\left((m+n) \mathbf{I}-\mathbf{F F}^{T}\right) .
$$

Then,

$$
\left\{\begin{array} { l } 
{ \frac { \partial \mathcal { L } ( \mathbf { F } ) } { \partial \mathbf { F } } = 0 } \\
{ \frac { \partial \mathcal { L } ( \mathbf { F } ) } { \partial \lambda } = 0 }
\end{array} \Rightarrow \left\{\begin{array}{l}
\mathbf{A} \mathbf{F}^{T}=\lambda \mathbf{F}^{T}, \\
\mathbf{I}=\frac{1}{(m+n)} \mathbf{F F}^{T} .
\end{array}\right.\right.
$$

Clearly, the optimization solution $\mathbf{F}=\left[\hat{\mathbf{z}}_{1}, \hat{\mathbf{z}}_{2}, \cdots, \hat{\mathbf{z}}_{d}\right]^{T}$ is formed by $d$ eigenvectors of $\mathbf{A}$ corresponding to the $d$ smallest nonzero eigenvalues of the eigenvalue problem:

$$
\mathbf{A} \hat{\mathbf{z}}_{i}=\lambda_{i} \hat{\mathbf{z}}_{i}
$$

where $\lambda_{i}$ is the eigenvalue and $\hat{\mathbf{z}}_{i}$ is the corresponding eigenvector. The whole SLLR method is summarized in Algorithm 1.

\section{Alignment performance evaluation: structural stability criteria}

In almost all alignment algorithms, performance is measured by the alignment accuracy. However, there are no evaluation metrics to evaluate the local structure preservation after manifold alignment. Based on the local structure similarity $[1,24]$, we propose the concept of structural stability to evaluate the local structure preservation. 


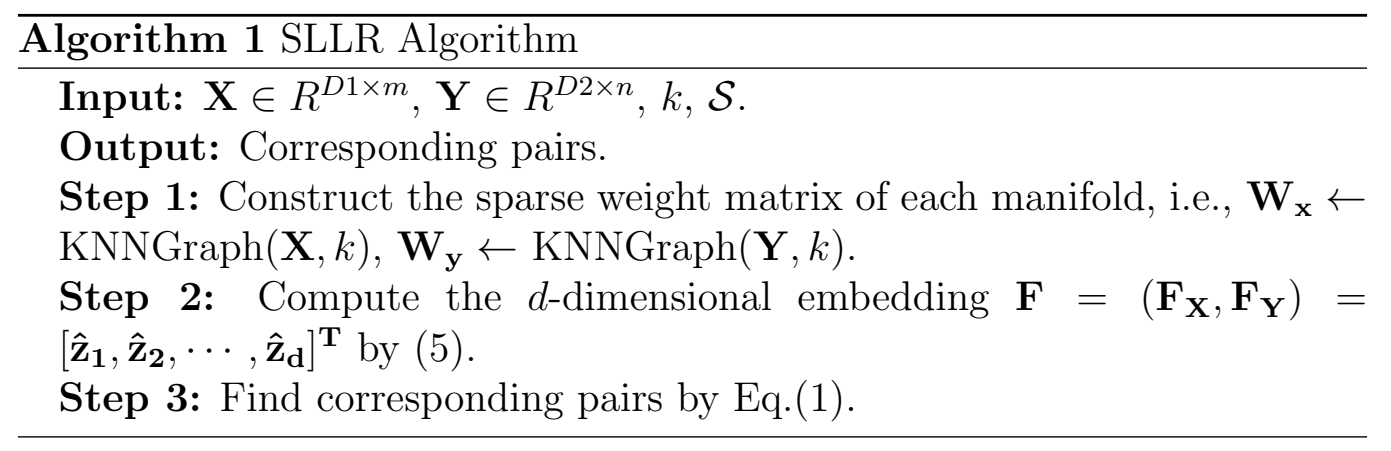

\subsection{Local Structure Similarity}

Given $\mathbf{X}$ and $\mathbf{Y}, \mathbf{F}_{\mathbf{X}}$ and $\mathbf{F}_{\mathbf{Y}}$ can be computed by alignment algorithms. Let $\mathbf{D}_{\mathbf{X}}$ and $\mathbf{D}_{\mathbf{Y}}$ denote the Euclidean distance matrices:

$$
\begin{gathered}
\left\{\begin{array}{l}
\mathbf{D}_{\mathbf{X}}=\left[d_{i j}^{\mathbf{x}}\right], \quad i=1,2, \cdots, m, j=1,2, \cdots, k, \\
\mathbf{D}_{\mathbf{Y}}=\left[d_{i j}^{\mathbf{y}}\right], \quad i=1,2, \cdots, n, j=1,2, \cdots, k,
\end{array}\right. \\
\left\{\begin{array}{l}
d_{i j}^{\mathbf{x}}=\left\|\mathbf{F}_{\mathbf{x}_{i}}-\mathbf{F}_{\mathbf{x}_{j}}\right\|_{2}^{2}, \text { and } d_{i 1}^{\mathbf{x}}<d_{i 2}^{\mathbf{x}}<\cdots<d_{i k}^{\mathbf{x}}, \\
d_{i j}^{\mathbf{y}}=\left\|\mathbf{F}_{\mathbf{y}_{i}}-\mathbf{F}_{\mathbf{y}_{j}}\right\|_{2}^{2}, \text { and } d_{i 1}^{\mathbf{y}}<d_{i 2}^{\mathbf{y}}<\cdots<d_{i k}^{\mathbf{y}},
\end{array}\right.
\end{gathered}
$$

where $d_{i j}^{\mathbf{x}}\left(d_{i j}^{\mathbf{y}}\right)$ describes the distance between the $i^{\text {th }}$ and $j^{\text {th }}$ points in the embedding space, and $k$ denotes the number of nearest neighbors. Each row vector $\mathbf{d}_{\mathbf{x}_{i}}=\left(d_{i 1}^{\mathbf{x}}, d_{i 2}^{\mathbf{x}}, \cdots, d_{i k}^{\mathbf{x}}\right)$ represents the relations between the $i^{t h}$ point and its nearest neighbors. These are used to characterize the local structure of in the embedding space.

To measure the local structure similarity between $\mathbf{F}_{\mathbf{x}_{i}}$ and $\mathbf{F}_{\mathbf{y}_{j}}$ in the embedding space, a parameterized curve representation is introduced. The discrete vector $\mathbf{d}_{\mathbf{x}_{i}}$ is fitted to the B-spline curve $g_{x_{i}}[24]$. There is a set of curves $G_{X}=\left\{g_{x_{i}} \mid i=1,2, \cdots, m\right\}, G_{Y}=\left\{g_{y_{j}} \mid j=1,2, \cdots, n\right\}$ (see Fig. $1(\mathrm{~b})$ ). The local structure similarity $\mathcal{A}_{i j}$ between $\mathbf{F}_{\mathbf{x}_{i}}$ and $\mathbf{F}_{\mathbf{y}_{j}}$ is measured based on enveloped areas and gradients [24]:

$$
\mathcal{A}_{i j}=\int_{0}^{k}\left(\left|g_{x_{i}}-g_{y_{j}}\right|+\beta\left|\nabla g_{x_{i}}-\nabla g_{y_{j}}\right|\right) d x
$$

where $\left|g_{x_{i}}-g_{y_{j}}\right|$ is the area enveloped by two curves, $\nabla g_{x_{i}}$ and $\nabla g_{y_{j}}$ are gradients of $g_{x_{i}}$ and $g_{y_{j}}$, respectively, and $\beta$ determines the trade-off between 
two terms. Thus

$$
\mathcal{A}=\left\{\mathcal{A}_{i j} \mid i=1,2, \cdots, m, j=1,2, \cdots, n\right\},
$$

which gives the structure similarity of any pairs in $\mathbf{F}_{\mathbf{X}}$ and $\mathbf{F}_{\mathbf{Y}}$ in the embedding space. Clearly, the higher the local structure similarity, the smaller the value of $\mathcal{A}_{i j}$. If $\left(\mathbf{F}_{\mathbf{x}_{i}} \leftrightarrow \mathbf{F}_{\mathbf{y}_{c}}\right), \mathcal{A}_{i c}$ should be the smallest entry of row vector $\mathcal{A}_{i}$.

\subsection{Structural Stability}

Definition 1. The structural stability is a fundamental property of an alignment algorithm, ensuring that the local structure of all existing corresponding pairs in the embedding space is unaffected by different sets of prior corresponding pairs with different cardinalities.

Suppose $\mathcal{C}=$ all existing corresponding pairs $\}$ between $\mathbf{F}_{\mathbf{X}}$ and $\mathbf{F}_{\mathbf{Y}}$. Given a set of prior corresponding pairs $\mathcal{S}$, let $\mathcal{N}_{s}$ denote the set of corresponding pairs found by an alignment algorithm with respect to $\mathcal{S}$. Clearly, $\mathcal{N}_{s} \subseteq \mathcal{C}$, with the subscript $s$ denoting the cardinality of the given set $\mathcal{S}$. $\operatorname{Card}\left(\mathcal{N}_{s}\right)$ indicates the cardinality of $\mathcal{N}_{s}$. Then, the alignment accuracy is determined as $\operatorname{Card}\left(\mathcal{N}_{s}\right) / \operatorname{Card}(\mathcal{C})$, where $\operatorname{Card}(\mathcal{S}) \leq \operatorname{Card}\left(\mathcal{N}_{s}\right) \leq \operatorname{Card}(\mathcal{C})$.

Given a set of prior corresponding pairs $\mathcal{S},\left(\mathbf{F}_{\mathbf{X}}, \mathbf{F}_{\mathbf{Y}}\right)$ and $\mathcal{N}_{s}$ can be found via alignment techniques such as Algorithm 1. Then, the structure similarity matrix $\mathcal{A}$ can be computed by Eq.(9). From (9), the structure similarity matrix $\mathcal{A}^{s} \in R^{m \times n}$ of $\mathcal{N}_{s}$ can be defined as

$$
\mathcal{A}^{s}\left\{\begin{aligned}
\mathcal{A}_{i j}^{s}= & \mathcal{A}_{i j} \quad \text { if }\left(\mathbf{F}_{\mathbf{x}_{i}} \leftrightarrow \mathbf{F}_{\mathbf{y}_{j}}\right) \in \mathcal{N}_{s} \\
& (i=1,2, \cdots, m ; j=1,2, \cdots, n), \\
\mathcal{A}_{i j}^{s}=0 \quad \text { otherwise } &
\end{aligned}\right.
$$

where the superscript $s$ denotes the cardinality of $\mathcal{S}$.

Given another set of prior corresponding pairs $\Theta$, where $\Theta$ is a proper superset of $\mathcal{S}$, i.e., $(\mathcal{S} \subset \Theta),\left(\mathbf{F}_{\mathbf{X}}, \mathbf{F}_{\mathbf{Y}}\right)$ and $\mathcal{N}_{\theta}$ can be found via alignment algorithms. Then, the structure similarity matrix $\mathcal{A}^{\theta} \in R^{m \times n}$ of $\mathcal{N}_{\theta}$ can be defined as

$$
\mathcal{A}^{\theta}\left\{\begin{aligned}
& \mathcal{A}_{i j}^{\theta}= \mathcal{A}_{i j} \quad \text { if }\left(\mathbf{F}_{\mathbf{x}_{i}} \leftrightarrow \mathbf{F}_{\mathbf{y}_{j}}\right) \in \mathcal{N}_{\theta} \\
&(i=1,2, \cdots, m ; j=1,2, \cdots, n), \\
& \mathcal{A}_{i j}^{\theta}=0 \quad \text { otherwise. }
\end{aligned}\right.
$$


Under different sets of prior corresponding pairs with different cardinalities, the structural invariant of corresponding pairs found by an alignment algorithm reflects the structural stability in the embedding space. This means that $\mathcal{A}_{i j}^{s}$ is similar to $\mathcal{A}_{i j}^{\theta}$ for $\left(\mathbf{F}_{\mathbf{x}_{i}} \leftrightarrow \mathbf{F}_{\mathbf{y}_{j}}\right)$.

Therefore, the change from $\mathcal{A}^{\theta=\operatorname{Card}(\mathcal{C})}$ to $\mathcal{A}^{s}$ can be used to quantify the structural stability of the algorithm-it reflects the change in local structures in the embedding space. That is to say, the structural difference is:

$$
\left\|\mathcal{A}^{\theta=\operatorname{Card}(\mathcal{C})}-\mathcal{A}^{s<\operatorname{Card}(\mathcal{C})}\right\|_{2},
$$

which is defined as the structural stability metric. As we can see, if the structural difference is small, the local structure of corresponding pairs is well preserved in the embedding space.

Remark 1. Consider $s<\operatorname{Card}(\mathcal{C})$. If the structural difference $\| \mathcal{A}^{\theta=\operatorname{Card}(\mathcal{C})}-$ $\mathcal{A}^{s<\operatorname{Card}(\mathcal{C})} \|_{2}$ is a small constant, then the local structure of corresponding pairs is unaffected by different sets of prior corresponding pairs with different cardinalities. Simultaneously, the set of corresponding pairs $\mathcal{N}_{s}$ can be found, and $\operatorname{Card}\left(\mathcal{N}_{s}\right)$ is approximately (or equal to) $\operatorname{Card}\left(\mathcal{N}_{\theta=\operatorname{Card}(\mathcal{C})}\right)$.

Remark 2. If the structural difference $\left\|\mathcal{A}^{\theta=\operatorname{Card}(\mathcal{C})}-\mathcal{A}^{s<\operatorname{Card}(\mathcal{C})}\right\|_{2}$ is a small constant, then the algorithm has good structural stability.

Therefore, it is easy to state the following:

Remark 3. If the structural stability of an algorithm is good, then the local structure of the data set can be preserved well in the embedding space.

Remark 4. If the structural stability of an algorithm is good, then Card $\left(\mathcal{N}_{s<\operatorname{Card}(\mathcal{C})}^{C}\right) / \operatorname{Card}(\mathcal{C}) \rightarrow 1$ when the set $S$ is small.

Clearly, the structural stability is a fundamental property of a manifold alignment algorithm. It can be seen that better structural stability in an algorithm will produce better performance in terms of local structure preservation and higher alignment accuracy. 


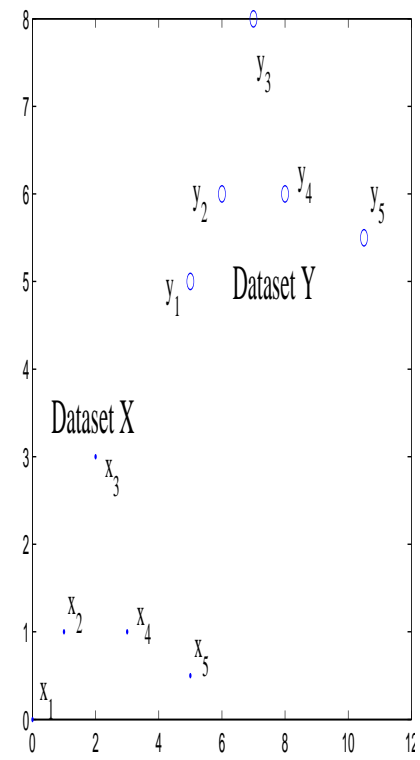

(a)
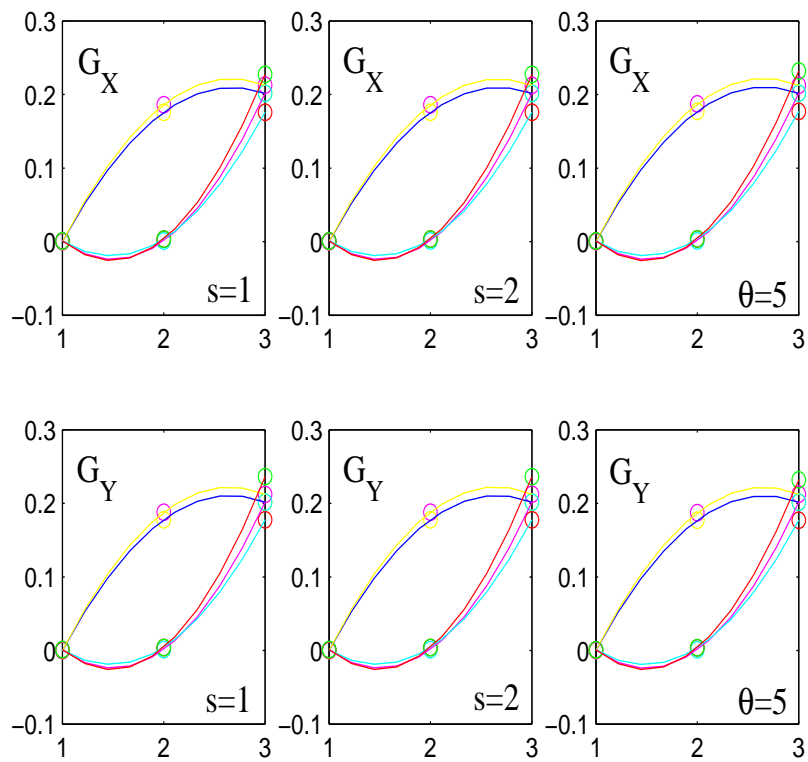

(b)

Figure 1: (a) Two synthetic datasets $\mathbf{X}=\left\{\mathbf{x}_{1}, \cdots, \mathbf{x}_{5}\right\} \in R^{2 \times 5}, \mathbf{Y}=\left\{\mathbf{y}_{1}, \cdots, \mathbf{y}_{5}\right\} \in R^{2 \times 5}$. (b) $G_{X}$ and $G_{Y}$ for different sets of prior corresponding pairs. Each subgraph has five curves in different colors to characterize the local structure of each point.

\subsection{A Simple Example}

Given two manifolds $\mathbf{X}$ and $\mathbf{Y}$ (shown in Fig. 1(a)), it can be seen that all corresponding pairs should be as follows: $\mathcal{C}=\left\{\left(\mathbf{x}_{1} \leftrightarrow \mathbf{y}_{1}\right),\left(\mathbf{x}_{2} \leftrightarrow\right.\right.$ $\left.\left.\mathbf{y}_{2}\right),\left(\mathbf{x}_{3} \leftrightarrow \mathbf{y}_{3}\right),\left(\mathbf{x}_{4} \leftrightarrow \mathbf{y}_{4}\right),\left(\mathbf{x}_{5} \leftrightarrow \mathbf{y}_{5}\right)\right\}$ and $\operatorname{Card}(\mathcal{C})=5$. For $\mathcal{S}=\mathcal{C}$, most alignment methods can determine all corresponding pairs and preserve the local structure of each manifold. However, if $\operatorname{Card}(\mathcal{S})<\operatorname{Card}(\mathcal{C})$, different algorithms will yield different results. The proposed method of Algorithm 1 is used in this example.

Under different sets of prior corresponding pairs, $\left(\mathbf{F}_{\mathbf{X}}, \mathbf{F}_{\mathbf{Y}}\right)$ and $\mathcal{N}_{s}$ can be found via Algorithm 1. The structure similarity matrix $\mathcal{A}^{s}$ of $\mathcal{N}_{s}$ can be computed by Eq.(10) or Eq.(11) (see Table 1). It holds that

$$
\left\{\begin{array}{l}
\left\|\mathcal{A}^{\theta=5}-\mathcal{A}^{s=1}\right\|_{2}=0.007 \\
\left\|\mathcal{A}^{\theta=5}-\mathcal{A}^{s=2}\right\|_{2}=0.007
\end{array}\right.
$$

which shows that the local structures of corresponding pairs change little with different sets of prior corresponding pairs. From the Remarks above, 
Table 1: The structure similarity matrix $\mathcal{A}^{s}$ of $\mathcal{N}_{s}$ corresponding to Fig. 1(b), and $\mathcal{A}_{i j}^{s}=0$ for $\left(\mathbf{x}_{i} \leftrightarrow \mathbf{y}_{j}\right) \notin \mathcal{N}_{s}$.

\begin{tabular}{lccccc}
\hline $\mathcal{A}^{s=1}$ & $\mathcal{A}_{11}^{s=1}$ & $\mathcal{A}_{22}^{s=1}$ & $\mathcal{A}_{33}^{s=1}$ & $\mathcal{A}_{44}^{s=1}$ & $\mathcal{A}_{55}^{s=1}$ \\
& 0.0030 & 0.0027 & 0.0008 & 0.0021 & 0.0054 \\
\hline $\mathcal{A}^{s=2}$ & $\mathcal{A}_{11}^{s=2}$ & $\mathcal{A}_{22}^{s=2}$ & $\mathcal{A}_{33}^{s=2}$ & $\mathcal{A}_{44}^{s=2}$ & $\mathcal{A}_{55}^{s=2}$ \\
& 0.0029 & 0.0030 & 0.0008 & 0.0020 & 0.0054 \\
\hline $\mathcal{A}^{\theta=5}$ & $\mathcal{A}_{11}^{\theta=5}$ & $\mathcal{A}_{22}^{\theta=5}$ & $\mathcal{A}_{33}^{\theta=5}$ & $\mathcal{A}_{44}^{\theta=5}$ & $\mathcal{A}_{55}^{\theta=5}$ \\
$1.0 \mathrm{e}-004^{*}$ & 0.2234 & 0.2289 & 0.1354 & 0.2665 & 0.8141 \\
\hline
\end{tabular}

Table 2: The alignment accuracy (\%) corresponding to Fig.1 (b)

\begin{tabular}{lccccc}
\hline METHOD & $s=1$ & $s=2$ & $s=3$ & $s=4$ & $\theta=5$ \\
\hline SLLR & 100 & 100 & 100 & 100 & 100 \\
\hline
\end{tabular}

we can conclude that the structural stability of the algorithm is good. The local structure of corresponding pairs is preserved well, as shown in Fig. 1(b). The corresponding alignment accuracy of the proposed algorithm is 100\% (see Table 2).

\section{Experiments}

In this section, we evaluate the performance of SLLR on a number of synthetic and real datasets. We manually tune the parameters of each method and report their best results with fixed parameters on different datasets. The following parameter values are used: $\nu=1, \mu=100$ in Wang's method [1], $\alpha_{x}=0.5, \alpha_{y}=0.5$ in MAP [41], and $\mu_{s}=10, \mu_{x}=5, \mu_{y}=5$ in our method, unless otherwise stated. Please refer to $[1,41]$ for details.

\subsection{Experiments with Synthetic Data}

The datasets in the example (see Fig. 1(a)) are used to illustrate the efficiency of the proposed method.

\subsubsection{The Role of Alignment: $\mathrm{E}_{\mathrm{s}}$ or $\left(\mathrm{E}_{\mathrm{x}}\right.$ and $\left.\mathrm{E}_{\mathrm{y}}\right)$ ?}

When the set of prior corresponding pairs $\mathcal{S}$ is empty, i.e., $s=0$, the loss function Eq.(2) can be rewritten as:

$$
\mathcal{L}(\mathbf{F})=\mu_{x} \mathbf{E}_{\mathbf{x}}+\mu_{y} \mathbf{E}_{\mathbf{y}}
$$



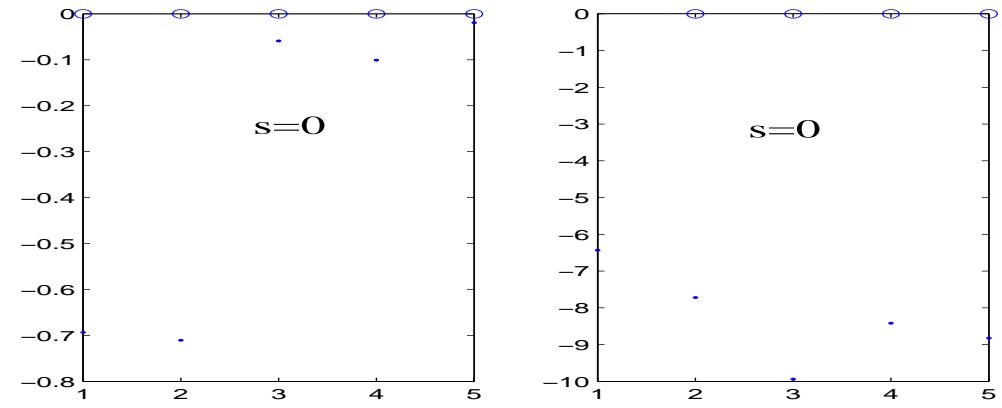

Figure 2: The left figure illustrates the alignment result given by our method, and the right figure illustrates the alignment result using Wang's method with $d=1$. In each subgraph, each circle/dot represents a point, plotted by order on the horizontal axis and coordinates on the vertical.

This can be viewed as a dimensionality reduction method. The alignment result of Eq.(13) is shown in Fig. 2. In each subgraph, the circles/dots represent points in low-dimensional space. As shown in Table 4, the alignment accuracy of both methods is zero when $s=0$. Combining the techniques in $[1,2,3]$, it can be concluded that most common dimensionality reduction methods cannot be directly used to align synthetic datasets, and the corresponding information plays an important role in manifold alignment.

\subsubsection{Alignment Results and Structural Stability}

For different sets of prior corresponding pairs with different cardinalities, Fig. 3 illustrates the alignment results of SLLR and Wang's method. The corresponding B-spline curves $G_{X}$ and $G_{Y}$ are shown in Fig. 1(b) and Fig. 4(a), respectively. Fig. 4(b) shows the B-spline curves given by MAP [41]. The structural differences between the three methods are shown in Table 3. The alignment accuracies are shown in Table 4. It is easy to see that the Bspline curves of SLLR change only slightly with the increase in the number of corresponding pairs $s \in\{1,2,5\}$, and the structural difference resulting from SLLR is the smallest. Thus, SLLR effectively preserves the local structure of corresponding pairs in the embedding space, and obtains high alignment accuracy.

In addition, Table 4 shows that the alignment accuracy of MAP only reaches $100 \%$ when $s=3$. However, the structural difference produced by MAP is high (see Table 3). Thus, high alignment accuracy does not necessarily imply good structural stability. 

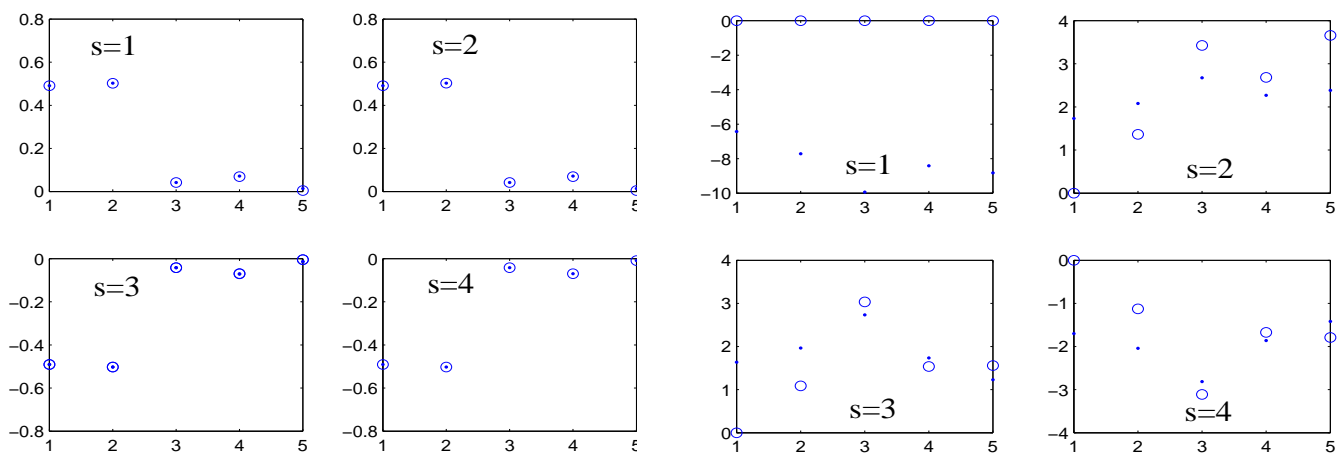

(a)

(b)

Figure 3: $d=1$, (a) the alignment result given by our method, (b) the alignment result using Wang's method.

Table 3: The Structural Stability $(\mathcal{S S})$ corresponding to Fig. 3 and the best $\mathcal{S} \mathcal{S}$ given by MAP, and the maximal alignment accuracy can be got (see Table 4).

\begin{tabular}{lcc}
\hline & $\left\|\mathcal{A}^{\theta=5}-\mathcal{A}^{s=1}\right\|_{2}$ & $\left\|\mathcal{A}^{\theta=5}-\mathcal{A}^{s=2}\right\|_{2}$ \\
\hline SLLR & 0.007 & 0.007 \\
WANG & 5.23 & 10.18 \\
MAP & 122.27 & 34.60 \\
\hline
\end{tabular}

Table 4: The maximal and mean alignment accuracies (\%) of the three methods with $\alpha_{x}=0.05, \alpha_{y}=0.05$

\begin{tabular}{l|c|ccccc}
\hline METHOD & $s=0$ & $\mathrm{ACC}$ & $s=1$ & $s=2$ & $s=3$ & $s=4$ \\
\hline \multirow{2}{*}{ MAP } & \multirow{2}{*}{0} & $\max$ & 60.00 & 80.00 & 100 & 80.00 \\
& & $\operatorname{mean}$ & 42.50 & 62.10 & 68.60 & 76.20 \\
\hline \multirow{2}{*}{ Wang } & \multirow{2}{*}{0} & $\max$ & 40 & 60 & 80 & 80 \\
& & $\operatorname{mean}$ & 31.93 & 51.86 & 57.66 & 64.19 \\
\hline \multirow{2}{*}{ SLLR } & \multirow{2}{*}{0} & $\max$ & 100 & 100 & 100 & 100 \\
& & mean & 88.83 & 98.3 & 100 & 100 \\
\hline
\end{tabular}



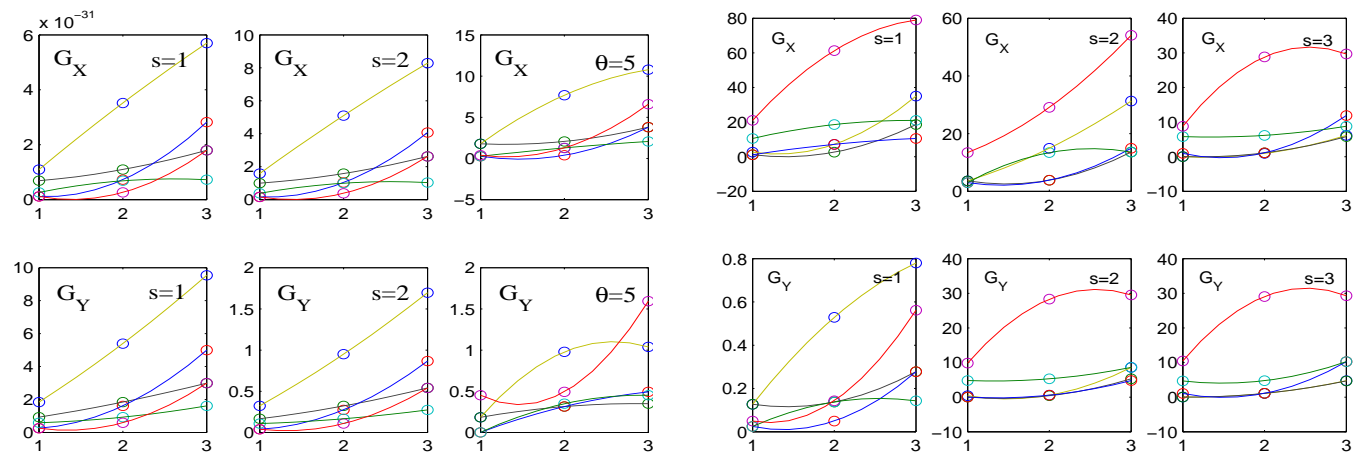

(a)
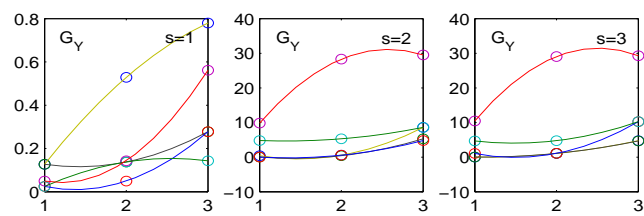

(b)

Figure 4: For different sets of prior corresponding pairs, (a) $G_{X}$ and $G_{Y}$ using Wang's method, (b) $G_{X}$ and $G_{Y}$ given by MAP. Each subgraph has five curves in different colors to characterize the local structure of each point.

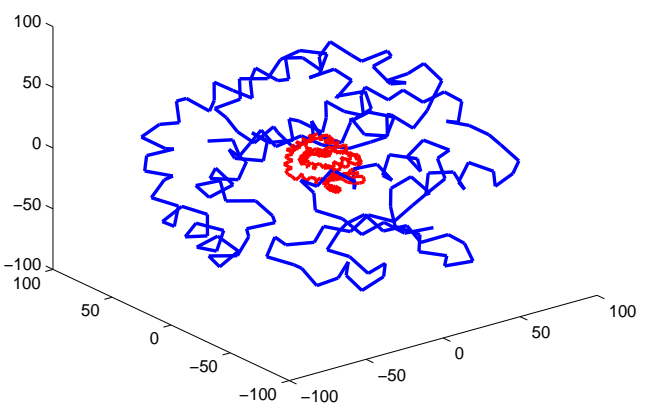

(a)
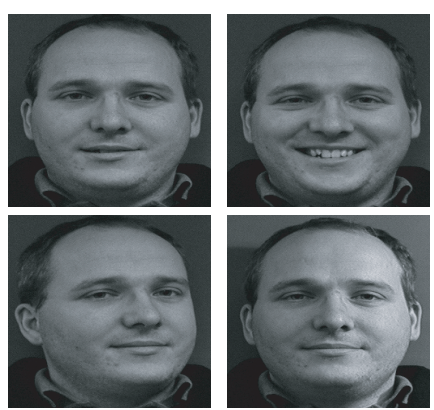

(b)

Figure 5: (a) the $1^{\text {st }}$ and $21^{\text {st }}$ protein models. (b) example figures from the IMM face datebase. 

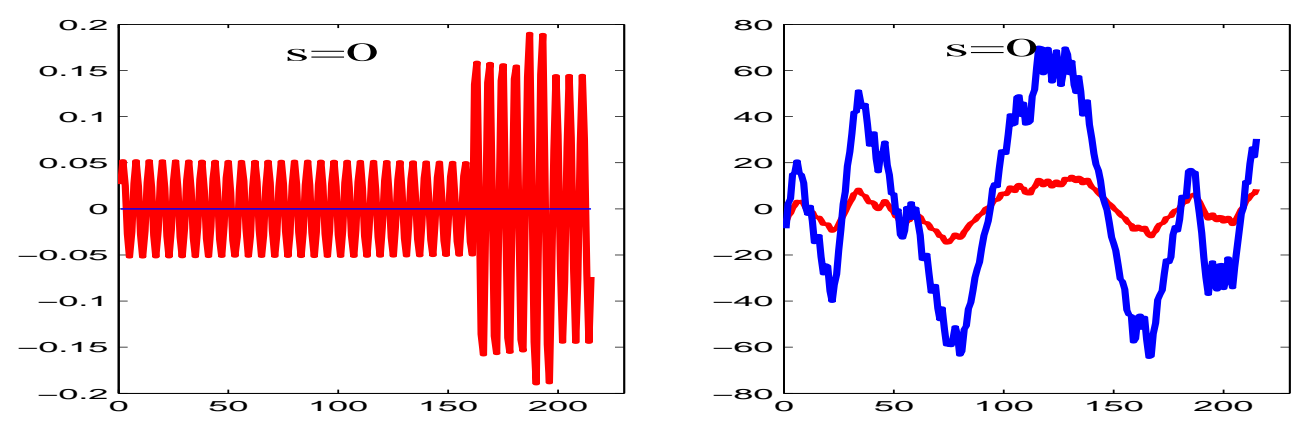

Figure 6: The left figure illustrates the alignment result using our method, and the right figure illustrates that from Wang's method with $d=1$.

\subsection{Experiments With Real Data: PDB-1G7O}

Analysis of protein structures can lead to a deep insight into their functions. Alignment experiments are performed on the $1^{\text {st }}$ and $21^{\text {st }}$ protein models from the Glutaredoxin protein structure database PDB-1G7O [42]. Each model has 215 amino acids and is represented in $3 D$ space (see Fig. $5(\mathrm{a}))$.

\subsubsection{The Role of Alignment: $\mathrm{E}_{\mathrm{s}}$ or $\left(\mathrm{E}_{\mathrm{x}}\right.$ and $\left.\mathrm{E}_{\mathrm{y}}\right)$ ?}

When $\mathcal{S}$ is the empty set, i.e., $s=0$, the loss function Eq.(2) can be rewritten as Eq.(13). This can be viewed as a dimensional reduction of the two protein models. The alignment results given by Eq.(13) are shown in Fig. 6. In each subgraph, ${ }^{-}{ }^{\prime}$ represents a point in low-dimensional space. As shown in Table 6 , the alignment accuracy of both methods is approximately zero when $s=0$. Combining the techniques in $[1,2,3]$, it can be concluded that most common dimensionality reduction methods cannot be directly used to align real datasets, and the corresponding information plays an important role in manifold alignment.

\subsubsection{Alignment Results and Structural Stability}

Under different sets of prior corresponding pairs with different cardinalities, Fig. 7 illustrates the alignment results of SLLR and Wang's method. The structural differences between the three methods are shown in Table 5, and the alignment accuracies are shown in Table 6 . It is easy to see that the structural difference produced by SLLR is the smallest. Thus, using SLLR, the local structure of corresponding pairs is preserved well in the embedding space, and high alignment accuracy is obtained. 

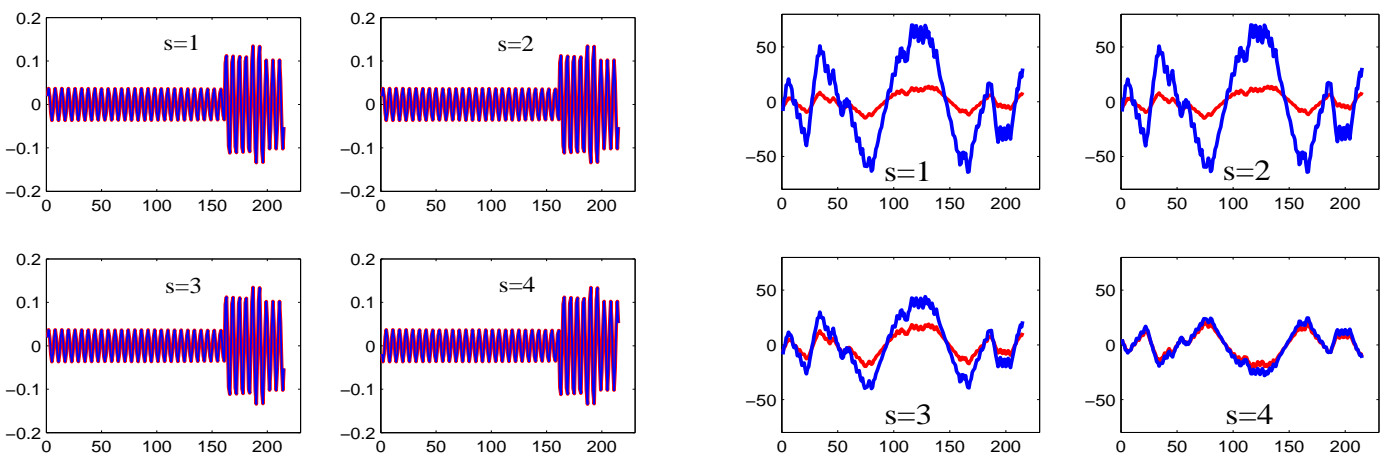

(a)

(b)

Figure 7: For different sets of prior corresponding pairs, (a) the alignment result given by our method, (b) the alignment result using Wang's method.

Table 5: The corresponding structural stability $(\mathcal{S S})$ in figure 7 and the best $\mathcal{S} \mathcal{S}$ given by MAP, and the maximal alignment accuracy can be got (see Table 6 ).

\begin{tabular}{lcc}
\hline & $\left\|\mathcal{A}^{\theta=215}-\mathcal{A}^{s=1}\right\|_{2}$ & $\left\|\mathcal{A}^{\theta=215}-\mathcal{A}^{s=2}\right\|_{2}$ \\
\hline SLLR & 0.0 & 0.0 \\
Wang & 13.44 & 3.60 \\
MAP & 187.69 & 230.74 \\
\hline
\end{tabular}

Table 6: The maximal and mean alignment accuracies (\%) of the three methods with $k=2$.

\begin{tabular}{l|l|ccccc}
\hline METHOD & $s=0$ & $\mathrm{ACC}$ & $s=1$ & $s=2$ & $s=3$ & $s=4$ \\
\hline \multirow{2}{*}{ MAP } & \multirow{2}{*}{0} & $\max$ & 2 & 3.25 & 5.5 & 6.9 \\
& & $\operatorname{mean}$ & 1 & 1.1 & 1.3 & 1.6 \\
\hline \multirow{2}{*}{ Wang } & \multirow{2}{*}{0.01} & $\max$ & 16.74 & 26.04 & 28.83 & 30.69 \\
& & $\operatorname{mean}$ & 1 & 4 & 7 & 9 \\
\hline \multirow{2}{*}{ SLLR } & \multirow{2}{*}{0.00} & $\max$ & 100 & 100 & 100 & 100 \\
& & mean & 100 & 100 & 100 & 100 \\
\hline
\end{tabular}



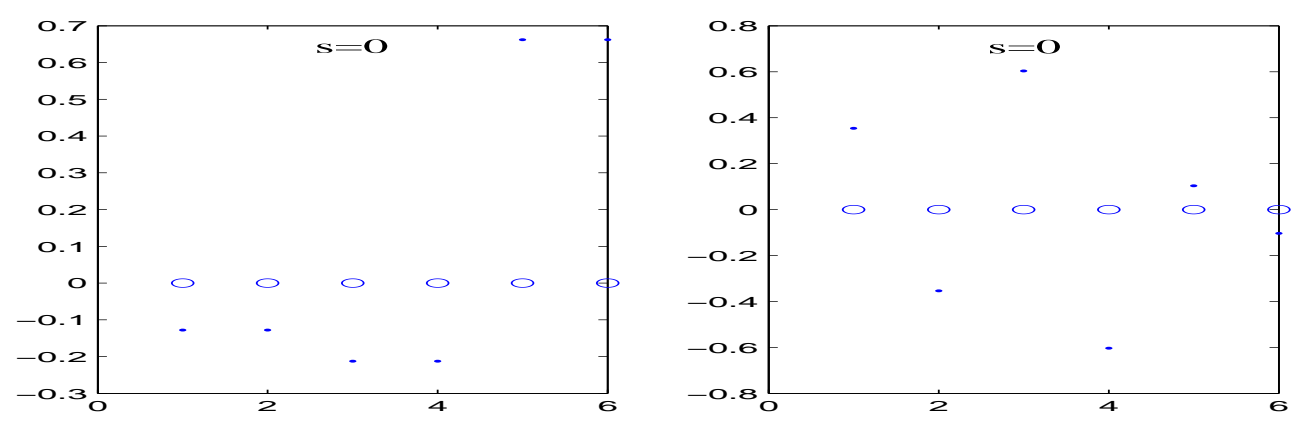

Figure 8: The left figure illustrates the alignment result from our method, and the right figure illustrates the alignment result given by Wang's method.
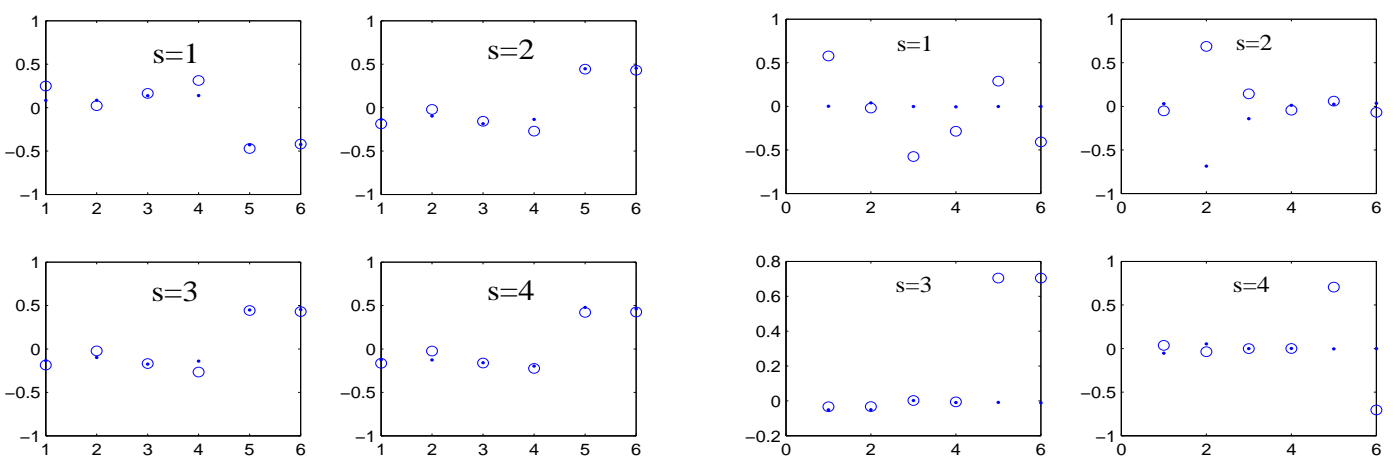

(a)

(b)

Figure 9: Under different sets of prior corresponding pairs, (a) the alignment result given by SLLR, (b) the alignment result using Wang's method.

\subsection{Experiments with Real Data: IMM Face}

The IMM Face Database comprises 240 still images of 40 different human faces [43]. There are six images of each person, captured with a fixed camera and exhibiting various poses and facial expressions (see Fig. 5(b)). Compared with the COIL-20 database, which only contains variations in pose [44], this presents the challenge of aligning images with multiple variables.

\subsubsection{The Role of Alignment: $\mathrm{E}_{\mathrm{s}}$ or $\left(\mathrm{E}_{\mathrm{x}}\right.$ and $\left.\mathrm{E}_{\mathrm{y}}\right)$ ?}

The alignment results given by Eq.(13) are shown in Fig.8. In each subgraph, the circles/dots represent points in low-dimensional space. Again, we can conclude that most common dimensionality reduction methods cannot be directly used to align real datasets. 
Table 7: The corresponding structural stability $(\mathcal{S S})$ in figure 9 and the best $\mathcal{S} \mathcal{S}$ given by MAP, and the maximal alignment accuracy can be got (see Table 8).

\begin{tabular}{lcc}
\hline & $\left\|\mathcal{A}^{\theta=6}-\mathcal{A}^{s=1}\right\|_{2}$ & $\left\|\mathcal{A}^{\theta=6}-\mathcal{A}^{s=2}\right\|_{2}$ \\
\hline SLLR & 0.9034 & 0.8307 \\
Wang & 2.6222 & 1.6685 \\
MAP & 4.2960 & 5.0279 \\
\hline
\end{tabular}

Table 8: The maximal and mean alignment accuracies (\%) of the three methods with $k=4$ and $\mu=2$.

\begin{tabular}{l|c|ccccc}
\hline METHOD & $s=0$ & $\mathrm{ACC}$ & $s=1$ & $s=2$ & $s=3$ & $s=4$ \\
\hline \multirow{2}{*}{ MAP } & \multirow{2}{*}{16.67} & $\max$ & 33.33 & 50.00 & 66.67 & 100 \\
& & $\operatorname{mean}$ & 31.67 & 39.16 & 56.67 & 71.67 \\
\hline \multirow{2}{*}{ Wang } & \multirow{2}{*}{16.67} & $\max$ & 66.67 & 66.67 & 83.33 & 100 \\
& & $\operatorname{mean}$ & 37.91 & 38.50 & 55.83 & 62.33 \\
\hline \multirow{2}{*}{ SLLR } & \multirow{2}{*}{33.33} & $\max$ & 66.67 & 83.33 & 83.33 & 100 \\
& & mean & 49.16 & 64.16 & 75.83 & 77.50 \\
\hline
\end{tabular}

\subsubsection{Alignment Results and Structural Stability}

Under different sets of prior corresponding pairs with different cardinalities, Fig.9 illustrates the alignment results given by SLLR and Wang's method. The structural differences between the three methods are shown in Table 7, and the alignment accuracies are shown in Table 8. It is easy to see that SLLR produces the smallest structural difference. Thus, using SLLR, the local structure of corresponding pairs is preserved well in the embedding space, and high alignment accuracy is obtained.

\section{Conclusions}

This paper proposed a new semi-supervised alignment method based on local structure preservation in each manifold. The shared intrinsic structure is obtained by formulating an optimization problem with a closed-form solution. This simultaneously matches the corresponding instances and preserves the local geometry of each manifold. Furthermore, the concept of structural stability was proposed to evaluate the local structure preservation. It has been shown that better structural stability leads to higher alignment accuracy, but the converse was shown not to hold. Benefiting from the locally linear reconstruction in each manifold, our method exhibits much better 
structural stability and higher alignment accuracy. Experimental results using both synthetic and real datasets demonstrate that our method achieves better performance than other related alignment methods.

\section{Acknowledgment}

This work was supported by the National Science Foundation of China (Grant No. 61375065, 61502208 and 61602066), partially supported by the State Key Program of National Science Foundation of China (Grant No. 61432012 and 61432014), and by the Project Supported by the Scientific Research Foundation (KYTZ201608) of CUIT, and by the Sichuan Science and Technology Support Project under Grant No.2014SZ0104, and by the Natural Science Foundation of the Jiangsu Higher Education Institutions of China (Grant No.14KJB520007), China Postdoctoral Science Foundation (Grant No. 2015 M570411).

[1] C. Wang, P. Krafft, S. Mahadevan, Manifold alignment, IEEE Trans. on Siganl Processsing 51 (3) (2011) 839-852.

[2] C. Wang, S. Mahadevan, Manifold alignment using procrustes analysis, in: International Conference on Machine Learning (ICML), 2008, pp. $1120-1127$.

[3] J. Ham, D. Lee, L. Saul, Semisupervised alignment of manifolds, in: Proceedings of the Annual Conference on Uncertainty in Artificial Intelligence, Z. Ghahramani and R. Cowell, Eds, Vol. 10, 2005, pp. 120-127.

[4] E. Elhamifar, R. Vidal, Sparse manifold clustering and embedding, in: Advances in neural information processing systems, 2011, pp. 55-63.

[5] M. Wang, Y. Gao, K. Lu, Y. Rui, View-based discriminative probabilistic modeling for $3 \mathrm{~d}$ object retrieval and recognition, IEEE Transactions on Image Processing 22 (4) (2013) 1395-1407. doi:10.1109/TIP.2012.2231088.

[6] J. Yu, R. Hong, M. Wang, J. You, Image clustering based on sparse patch alignment framework, Pattern Recognition 47 (11) (2014) 3512 3519 . 
[7] M. Wang, X. Liu, X. Wu, Visual classification by 11 hypergraph modeling, IEEE Transactions on Knowledge and Data Engineering 27 (9) (2015) 2564-2574. doi:10.1109/TKDE.2015.2415497.

[8] R. N. Shepard, Multidimensional scaling, tree-fitting, and clustering, Science, New Series 210 (4468) (1980) 390-398.

[9] M. Wang, W. Fu, S. Hao, D. Tao, X. Wu, Scalable semi-supervised learning by efficient anchor graph regularization, IEEE Transactions on Knowledge and Data Engineering 28 (7) (2016) 1864-1877.

[10] J. Tenenbaum, V. Silva, J. Langford, A global geometric framework for nonlinear dimensionality reduction, Science 290 (5500) (2000) 2319 2323.

[11] S. Roweis, L. Saul, Nonlinear dimensionality reduction by local linear embedding, Science 290 (5500) (2000) 2323-2326.

[12] Y. Fu, T. S. Huang, Locally linear embedded eigenspace analysis, IFPTR, Univ. of Illinois at Urbana-Champaign.

[13] M. Belkin, P. Niyogi, Laplacian eigenmaps for dimensionality reduction and data representation, Neural Comput. 15 (6) (2003) 1373-1396.

[14] J. C. Lv, K. K. Tan, Z. Yi, S. Huang, A family of fuzzy learning algorithms for robust principal component analysis neural networks, IEEE Trans. on Fuzzy Systems 18 (1) (2010) 217 -226.

[15] J. C. Lv, Z. Yi, J. Zhou, Subspace Learning of Neural Networks, CRC press, CRC, 2011.

[16] J. C. Lv, Z. Yi, K. K. Tan, Global convergence of oja's pca learning algorithm with a non-zero-approaching adaptive learning rate, Theoretical computer science 367 (3) (2006) 286-307.

[17] X. He, P. Niyogi, Locality preserving projections, in: NIPS, Vol. 16, 2003, pp. 234-241.

[18] Y. Xu, A. Zhong, J. Yang, D. Zhang, \{LPP $\}$ solution schemes for use with face recognition, Pattern Recognition 43 (12) (2010) 4165 - 4176. 
[19] J. Yu, Y. Guo, D. Tao, J. Wan, Human pose recovery by supervised spectral embedding, Neurocomputing 166 (2015) 301 - 308.

[20] H. T. Vu, C. Carey, S. Mahadevan, Manifold warping: Manifold alignment over time., in: AAAI, 2012, p. 8.

[21] P. Viola, W. M. Wells III, Alignment by maximization of mutual information, in: Proceedings: Fifth International Conference on Computer Vision (ICCV), IEEE, 1995, pp. 16-23.

[22] C. Wang, S. Mahadevan, Manifold alignment without correspondence, in: Proceedings of the 21st International Joint Conference on Artifical Intelligence (IJCAI), Morgan Kaufmann Publishers Inc., San Francisco, 2009, pp. 1273-1278.

[23] C. Wang, S. Mahadevan, Multiscale analysis of document corpora based on diffusion models, in: Proceedings of the 21st International Joint Conference on Artifical Intelligence (IJCAI), Morgan Kaufmann Publishers Inc., San Francisco, 2009, pp. 1592-1597.

[24] P. Yuru, F. Huang, F. Shi, H. Zha, Unsupervised image matching based on manifold alignment, IEEE Trans. on Pattern Analysis and Machine Intelligence (TPAMI) 34 (8) (2012) 1658 -1664.

[25] J. Verbeek, Learning nonlinear image manifolds by global alignment of local linear models, IEEE Trans. on Pattern Analysis and Machine Intelligence(TPAMI) 28 (8) (2006) 1236-1250.

[26] J. J. Verbeek, S. T. Roweis, N. Vlassis, et al., Non-linear cca and pca by alignment of local models, Advances in Neural Information Processing Systems (NIPS) 16 (2004) 297-304.

[27] C. Wang, S. Mahadevan, A general framework for manifold alignment, in: AAAI Fall Symposium on Manifold Learning and its Applications, 2009, pp. 53-58.

[28] X. Li, J. C. Lv, Z. Yi, Manifold alignment based on sparse local structures of more corresponding pairs, in: International Joint Conference on Artificial Intelligence (IJCAI), 2013, pp. 2862-2868. 
[29] J. Yu, M. Wang, D. Tao, Semisupervised multiview distance metric learning for cartoon synthesis, IEEE Transactions on Image Processing 21 (11) (2012) 4636-4648.

[30] J. Feng, L. Bo, Y. Hong-xun, L. Shao-hui, Manifold learning and manifold alignment based on coupled linear projections, CAAI Transactions on Intelligent Systems 5 (6) (2010) 476-481.

[31] C. Wang, B. Liu, H. Vu, S. Mahadevan, Sparse manifold alignment, University of Massachusetts Department of Computer Science Technical Report UM-CS-2012-030.

[32] C. Wang, S. Mahadevan, Manifold alignment preserving global geometry., in: Proceedings of the Twenty-Third International Joint Conference on Artificial Intelligence (IJCAI), 2013, pp. 1743-1749.

[33] L. Xiong, F. Wang, C. Zhang, Semi-definite manifold alignment, in: Machine Learning: ECML 2007, Springer, 2007, pp. 773-781.

[34] G. Jianping, Z. Yi, Locality-based discriminant neighborhood embedding, The Computer Journal 56 (9) (2013) 1063-1082.

[35] J. Yang, D. Zhang, J. y. Yang, B. Niu, Globally maximizing, locally minimizing: Unsupervised discriminant projection with applications to face and palm biometrics, IEEE Transactions on Pattern Analysis and Machine Intelligence 29 (4) (2007) 650-664.

[36] Z. P. Bažant, Structural stability, International Journal of Solids and Structures 37 (1) (2000) 55-67.

[37] Z. P. Bažant, L. Cedolin, Stability of structures: elastic, inelastic, fracture and damage theories, World Scientific, 2010.

[38] C. Pugh, M. M. Peixoto, Structural stability, Scholarpedia 3 (9) (2008) 4008.

[39] S. Yan, H. Wang, Semi-supervised learning by sparse representation., in: SDM, 2009, pp. 792-801.

[40] D. Bremner, E. Demaine, J. Erickson, J. Iacono, Langerman, Outputsensitive algorithms for computing nearest-neighbour decision boundaries, Discrete \& computational geometry 33 (4) (2005) 593-604. 
[41] D. Zhai, H. Chang, L. Bo, S. Shiguang, C. Xilin, G. Wen, Manifold alignment via corresponding projections, BMVC (2010) 1-11.

[42] Z. M. J., C. Bystroff, Protein Structure Prediction Second Edition, Humana Press, Humana, 2008.

[43] M. B. Stegmann, B. K. Ersbøll, R. Larsen, FAME - a flexible appearance modelling environment, IEEE Trans. on Medical Imaging 22 (10) (2003) $1319-1331$.

[44] A. N. Samneer, S. K. Nayar, H. Murase, Coumbia object image library(coil-20), Department of Computer Science, Columbia University (1996) 1-4. 